\title{
Medial Rectus Recession Is as Effective as Lateral Rectus Resection in Divergence Paralysis Esotropia
}

\author{
Zia Chaudhuri, MS, FRCS (Glasg) and Joseph L. Demer, MD, PhD \\ Jules Stein Eye Institute, University of California, Los Angeles
}

\begin{abstract}
Objective-To propose medial rectus (MR) recession to be equally as effective as lateral rectus (LR) resection, which has heretofore been the preferred treatment for divergence paralysis esotropia (DPE).
\end{abstract}

Methods-We examined a 17-year surgical experience comparing LR resection with MR recession in adults with DPE, defined as symptomatic distance esotropia (ET) at least double the asymptomatic ET of 10 or less prism diopters $(\Delta)$ at near.

\begin{abstract}
Results-Twenty-four patients with DPE underwent surgery. Six patients underwent bilateral LR resection and 2 underwent unilateral LR resection (group L), while 13 underwent bilateral MR recession and 3 underwent unilateral MR recession, with the target angle double the distance ET (group M). One of 8 patients in group $\mathrm{L}$ and 15 of 16 patients in group $\mathrm{M}$ underwent intraoperative adjustable surgery under topical anesthesia. Mean (SD) preoperative central gaze ET measured 15.0 (7.7) $\Delta$ at distance and 4.1 (3.4) $\Delta$ at near in group L, but 10.4 (6.8) $\Delta$ at distance and 0.6 (1.7) $\Delta$ at near in group M ( $P=.15$; distance, 0.003 , near). Postoperatively, no patient in either group had symptomatic diplopia or convergence insufficiency in follow-up from 8.5 to 40 months. Twice the usual surgical dose of MR recession per prism diopter was required to achieve correction of the distance deviation in DPE as compared with that recommended for ET generally and also for LR resection in the same condition.
\end{abstract}

Conclusions-Recession of the MR provides binocular single vision in DPE without convergence insufficiency at near, and it is convenient for intraoperative adjustment under topical anesthesia.
Divergence paralysis esotropia (DPE)—variably termed divergence insufficiency, divergence insufficiency esotropia, and divergence paresis esotropia-is characterized by comitant esotropia (ET) at distance fixation associated with symptomatic diplopia, fusion at near fixation, and normal saccadic velocities in adduction and abduction. As the name implies, early thinking about DPE was dominated by the presumed pathophysiological concept of a brain lesion involving a putative divergence center. ${ }^{1,2}$ Indeed, isolated clinical reports have associated DPE with neurologic conditions such as brain tumors, multiple sclerosis, trauma, subdural hematoma, and tertiary syphilis. ${ }^{2-5}$ Even postulated as a cause of DPE was increased medial rectus (MR) muscle tonicity as a compensatory mechanism for

\footnotetext{
(C) 2012 American Medical Association

Correspondence: Joseph L. Demer, MD, PhD, Jules Stein Eye Institute, University of California, Los Angeles, 100 Stein Plaza, Los Angeles, CA 90095-7002 (jld@ucla.edu).

Author Contributions: Both authors had full access to all the data in the study and take responsibility for the integrity of the data and the accuracy of the data analysis.

Financial Disclosure: None reported.

Disclaimer: Magnetic resonance imaging surface coils used in this project are not Food and Drug Administration-approved for this purpose.
} 
presumed decreased age-related convergence ${ }^{6}$ and subclinical or barely clinical MR restriction. ${ }^{7}$ However, probably because most cases of DPE were not associated with obvious neurologic diseases or diminished convergence, an alternative proposition of mild bilateral abduction weakness was suggested. ${ }^{3,4,6}$ Most recently, high-resolution magnetic resonance imaging of the orbit has demonstrated inferior sagging of the lateral rectus (LR) pulley due to degeneration of the connective tissue of its suspensory ligament, the LR-SR band, in patients with DPE. ${ }^{8}$ Therefore, it has most recently been proposed that DPE is more typically a mechanical rather than a neurogenic disorder. Although based on the modern proposal for etiology, the term DPE has become a misnomer; it is used here as a convenience for reference to earlier literature and for clinical application.

The horizontal, binocular diplopia during distance viewing can be functionally significant in DPE, significantly impairing patients' quality of life. Surgical and nonsurgical treatments for

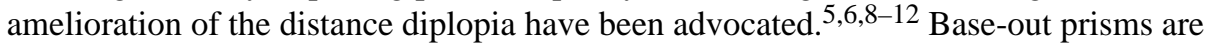
frequently effective, particularly for smaller deviations in patients already requiring spectacle correction for refractive errors. However, there is increasing motivation for spectacle independence among patients of all ages, particularly contact lens wearers, and patients who have undergone cataract surgery with implantation of multifocal intraocular lenses. In patients with DPE who do not desire prismatic correction or who have deviations exceeding reasonable spectacle prism power, resection of 1 or both LR muscles has been reported as effective. ${ }^{5,8-12}$ Preference for LR resection has been founded on the traditional assumption that the LR muscle is more important during distance fixation and the MR more important at near. However, the traditional assumption has more recently been challenged by both biomechanical modeling ${ }^{13,14}$ and surgical experience, ${ }^{7,15}$ indicating that MR recession would also be effective in DPE. At our institution, both LR resection and MR recession have been used for treatment of DPE, permitting comparison of the long-term effectiveness and complications of both surgical approaches.

\section{METHODS}

Under a protocol approved by the institutional review board of the Office for Protection of Human Subjects, a retrospective analysis of the clinical and surgical records of consecutive patients with DPE from 1994 through 2011 at the Pediatric Ophthalmology and Strabismus Division at the Jules Stein Eye Institute was performed. Divergence paralysis esotropia was stringently defined as orthophoria or asymptomatic esophoria of no more than $10 \Delta$ at 33 $\mathrm{cm}$, with symptomatic distance ET measuring at least twice the measured near esophoria.

Patients with DPE were divided into 2 groups, depending on the corrective surgical procedure performed: group L with LR resection and group $\mathrm{M}$ with MR recession. Intergroup comparisons were performed for age, sex, ductions in secondary and tertiary gazes, best-corrected visual acuity denoted as logarithm of the minimum angle of resolution ( $\log$ MAR), spherical equivalent refractive error, stereopsis (Titmus fly stereotest), preoperative deviation at distance and near, preoperative convergence amplitudes measured with base-out free prisms, amount of strabismus surgery performed, and postoperative binocular alignment recorded at multiple intervals until last follow-up.

Although never confirmed, it was supposed that a surgical exoshift exceeding the fusional convergence amplitude at near might have the undesirable effect of inducing convergence insufficiency. Consequently, convergence amplitudes were measured preoperatively for distance and near by progressively increasing base-out prism until diplopia was reported and alternate cover testing demonstrated induced exotropia. Therefore, surgically induced exoshift was never planned to exceed the fusional convergence amplitude at near. It was confirmed in all patients that base-out prism corrected the distance ET and alleviated the 
diplopia. Divergence amplitudes were not measured in most cases, as these were presumed to have been insufficient to compensate for the ET for which all of the patients presented.

All patients underwent strabismus surgery, with either intraoperative adjustment under monitored topical anesthesia or postoperative adjustment within 24 hours of surgery that had been performed under general anesthesia. Adjustment was usually performed in 1 eye, even if surgery was bilateral, although surgeons usually chose bilateral surgery.

Patients were considered eligible for surgery under topical anesthesia if they tolerated moderate preoperative pressure over the bulbar conjunctiva overlying the MR of 1 eye applied using a cotton swab stick in the office after topical application of $0.5 \%$ proparacaine hydrochloride. Strabismus surgery with intraoperative adjustment was performed using topical anesthesia by $1 \%$ lidocaine hydrochloride solution. An anesthesiologist monitored the procedure and on surgeon request, provided individually selected, minimal doses of propofol or alfentanil hydrochloride so that patients remained, or quickly became, alert and cooperative during intraoperative suture adjustment. ${ }^{16-18}$ Adjustment was performed in the seated upright position with any required refractive correction, with centered and horizontally eccentric targets at near and distance. Patients were considered to have had adjustable sutures if at least 1 adjustable suture was placed, regardless of whether the muscle position was changed at the time the suture was made permanent.

Statistical analysis was performed using the Microsoft Excel package and $t$ test. To account for the large number of comparisons, a level of 0.005 was required to demonstrate statistical significance. Regression analysis of the dosage of surgery in millimeters per prism diopter of distance deviation corrected by surgery was calculated for both subject groups using JMP statistical software (SAS Institute) and compared with the recommended surgical dosage according to Parks et al. ${ }^{19}$

\section{RESULTS}

Of the 24 patients with DPE, 8 ( 2 male and 6 female) underwent LR resection (group L: 6 bilateral, 2 unilateral). The remaining 16 patients (group M: 4 male and 12 female) underwent MR recession (13 bilateral, 3 unilateral). The average (SD) age of patients in group L was 75 (6) years vs 68 (14) years in group M ( $P=.22)$. Average (SD) visual acuity of patients in group L was $0.18(0.25) \log$ MAR vs $0.16(0.17) \operatorname{logMAR}$ in group M ( $P=.69)$. Mean (SE) refractive error was -2.62 (3.87) diopters (D) in group L vs -1.55 (2.65) $\mathrm{D}$ in group M $(P=.27)$. Average (SD) stereo threshold was 55 (28) $\operatorname{arcsec}$ in group L and 131 (140) arcsec in group $\mathrm{M}(P=.15)$. Mean (SD) preoperative central gaze ET for distance was 15.0 (7.7) $\Delta$ in group $\mathrm{L}$ and 10.4 (6.8) $\Delta$ in group $\mathrm{M}(P=.15)$. Mean (SD) preoperative central gaze esophoria at near was 4.1 (3.4) $\Delta$ in group L and 0.6 (1.7) $\Delta$ in group M; this difference was statistically $(P=.003)$ but probably not clinically significant. Convergence amplitudes (SD) for distance and near were recorded in only 2 patients in group $\mathrm{L}$, averaging 18 (4) $\Delta$ for both distance and near. Average (SD) convergence amplitudes of 14 patients in group $M$ were 20 (9) $\Delta$ for near and 19 (8) $\Delta$ for distance.

Seven of 8 patients in group $\mathrm{L}$ required general anesthesia and underwent postoperative adjustable suture technique, while 15 of 16 patients in group $M$ underwent surgery under topical anesthesia with intraoperative adjustable suture technique. The only patient in group $M$ who was not operated on under topical anesthesia had intense nystagmus precluding intraoperative adjustable surgery; no adjustable suture was placed in this patient. Bilateral LR resection was performed in 5 patients in group L, with unilateral surgery in 3 patients. In group $\mathrm{M}, 13$ of 16 patients underwent bilateral surgery, with 3 patients undergoing unilateral surgery. Nine of 13 patients in group M underwent symmetrical MR recessions, while the 
remaining 4 underwent asymmetrical surgery dependent on the preoperative lateral incomitance. The average (SD) LR resection performed was 4.5 (1.2) $\mathrm{mm}$ in group L; details of the amounts of adjustment performed were unavailable from the medical records. The average (SD) MR recession in group M was $3.7(0.7) \mathrm{mm}$, measured using calipers at the conclusion of intraoperative adjustment.

In all cases in both groups, there was satisfactory postoperative improvement in binocular alignment in primary gaze for distance, with complete correction of distance diplopia in central gaze with initial operation. The Figure demonstrates the relationship between total surgical dose, representing the sum of the right and left eye recession or resection in both groups. Linear regression was performed to relate effect to total surgical dosage with comparison with recommended surgical dosage according to Parks et al. ${ }^{19}$ The regression slopes indicate the incremental effect represented in prism diopter per millimeter of right plus left eye LR resection vs MR resection. Note in the Figure that the observed regression slope for LR resection of $2.4938 \Delta / \mathrm{mm}(R=0.92 ; P=.001)$ corresponds to the slope of 2.50 computed from the recommended surgical dosage of Parks et al for resection. ${ }^{19}$ Interestingly, while the observed regression slope of $2.5057 \Delta / \mathrm{mm}(R=0.67 ; P=.005)$ for MR recession was also nearly identical to both the observed and recommended slopes for LR resection, the observed slope for MR recession was almost exactly half the slope of the recommended surgical dosage according to Parks et al. ${ }^{19}$

When lateral incomitance was present, the adjustable suture was generally placed on the MR opposite the horizontal direction of the greatest ET; this often resulted in slightly greater recession of this MR than of its fellow. Thus, if the ET was greater on right than left gaze, the left MR was recessed on adjustable suture to obtain a greater effect in right gaze. In one unilaterally operated on case in group $\mathrm{M}$, lateral gaze diplopia was relieved only in central gaze and contralateral to the operated eye, necessitating later recession of the other MR to alleviate symptom.

In all cases in both groups, central gaze ET was eliminated with amelioration of distance diplopia and no induction of near diplopia in any case. Average alignment results for the 2 groups did not differ by amounts that were statistically or clinically significant (Table 1). In the final postoperative assessment, 4 patients in group $L$ had small angles of consecutive near exophoria averaging 1.3 (2.1) $\Delta$ for distance and 1.5 (1.8) $\Delta$ for near. However, none of the patients in either group had postoperative diplopia at near or distance or required prismatic correction. In group $\mathrm{M}$, the preoperative near deviation was very small and became nil postoperatively. There was no consecutive exophoria in any patient in group M.

\section{COMMENT}

Surgical treatment of small-angle strabismus must carefully avoid overcorrection, a complex consideration if there is incomitance among distance, near, and lateral gazes as is typical of DPE. Historically, the description of DPE has been muddled by the inclusion of varied definitions and etiologies and sometimes severe neurologic or orbital insults largely appropriate for nonsurgical management. ${ }^{1,6,20,21}$ During the past 2 decades, specific efforts have been made to clarify the pathogenesis of DPE and refine surgical correction.

Surgical correction of severe ET due to DPE and marked inferior displacement of the LR has concentrated on the etiology of the condition as demonstrated by orbital imaging. $8,22,23$ The procedures reported have included suture anastomosis of the LR and superior rectus (SR) muscle margins approximately $10 \mathrm{~mm}$ posterior to their insertion, with superior transposition of the LR muscle insertion; ${ }^{8}$ suture anastomosis of the margins of the LR and SR pulleys $;{ }^{8}$ loop myopexy approximating the LR and SR muscles without splitting with MR recession or scleral fixation; ${ }^{10,24}$ union of the LR and SR bellies after splitting these 
muscles longitudinally, combined with MR recession; ${ }^{12} \mathrm{MR}$ recession and LR resection in 1 eye; ${ }^{25}$ and LR resections. ${ }^{5,9,11}$ These complex procedures are not required to treat DPE.

Adjustable LR resection has been reported effective in DPE, without causing consecutive exotropia at near. ${ }^{5,11}$ Though not specifically reported for DPE associated with connective tissue degeneration in elderly individuals, MR recession has been reported to be as effective as LR resection for correcting distance ET. ${ }^{7,15,26,27}$ In this report, adjustable LR resection with average (SD) surgical dose of 4.5 (1.2) $\mathrm{mm}$ was effective for correcting DPE and did not cause consecutive exotropia. Adjustable MR recession with an average (SD) dose of 3.7 (0.7) $\mathrm{mm}$ was found to be equally effective to LR resection. In addition to equal effectiveness, MR recession has the advantage of convenience for intraoperative adjustment under topical anesthesia. ${ }^{16-18}$

The surgical response of common forms of ET and exotropia to rectus muscle surgery has been reported as the amount of angle correction per millimeter of recession or resection. The data of Mojon ${ }^{28}$ indicates that horizontal rectus plication alters the horizontal angle by 1.76$2.88 \Delta / \mathrm{mm}$ in ET and $2.42-2.84 \Delta / \mathrm{mm}$ in exotropia. In abducens palsy, data from Esser et $\mathrm{al}^{29}$ indicate a 3.0-3.2 $\Delta / \mathrm{mm}$ reduction in ET for LR resection. The current study of DPE is quantitatively comparable, finding a $2.50 \Delta / \mathrm{mm}$ reduction in ET for both LR resection and MR recession. It is important to reiterate that this surgical effect is smaller than the $5 \Delta / \mathrm{mm}$ effect recommended for other types of ET by Parks et al (Table 2). ${ }^{19}$ The reason for the greater required MR recession for correction of DPE than other forms of ET is unknown at the present time but might be related to the sag of the MR pulley in older people who are susceptible to connective tissue degeneration. ${ }^{22}$ However, this does not explain why DPE responds in a quantitatively typical fashion to doses of LR resection recommended for ET generally.

This report presents stringent criteria for clinically defining DPE based on a 17-year retrospective study. It is proposed that the term DPE is a misnomer and is actually owing to degenerative inferior sag of the LR muscle that converts some of its abducting to infraducting force, perhaps in combination with LR elongation. The current data demonstrate that recession of the more stable MR muscle, physiological antagonist of the putatively sagging LR muscle, corrects ET at distance without inducing convergence insufficiency at near in DPE. Moreover, MR recession is reversible and convenient for intraoperative adjustment under topical anesthesia, and it is as effective as LR resection, both in this series and elsewhere. ${ }^{5,11}$ In DPE, where degeneration of the suspensory ligaments of the LR pulley is the probable cause of LR sag, ${ }^{8,20,22-24}$ it is arguably preferable to avoid inducing further sag, and perhaps future recurrence of DPE, by operating on the affected LR muscle.

Our study also specifically deals with the surgical management of DPE as a nonneurogenic entity in which LR force generation is unimpaired. The pathogenesis is presumed to be of the sagging eye syndrome, postulated as progressive, generalized orbital connective tissue degeneration, usually age related, in the absence of neurologic disease. ${ }^{8}$ Normal saccades exclude LR weakness in most cases of DPE, ${ }^{5}$ as was a required clinical criterion in all cases in the current series. Analysis of extraocular muscles by high-resolution orbital magnetic resonance imaging in sagging eye syndrome and heavy eye syndrome has suggested that inferior LR pulley heterotopia is the cause of the inferior LR sag in DPE. ${ }^{8,17,22,30}$ Sagging eye syndrome is probably the most common etiology of surgically correctable DPE. Serious neurologic causes of DPE are associated with other neurologic manifestations, including abducting saccade slowing, nystagmus, and associated cranial neuropathies. ${ }^{2-4}$ Patients with DPE due to connective tissue degeneration have good convergence amplitudes, thus minimizing the risk of symptomatic postoperative exotropia at near. 
Based on our current experience with DPE, we emphasize the importance of surgical success of preoperative assessment of the ET for distance, near, and lateral gazes, as well as confirmation of the adequacy of convergence amplitudes for near. Based on the present data, we recommend that the target angle for correction by MR recession be twice the distance ET measurement in the most affected horizontal gaze direction (Table 2), and that for laterally concomitant DPE, surgery be divided symmetrically between eyes to optimize comitance of the result. When lateral incomitance exists preoperatively, differential recessions are suggested to obtain the greatest lateral effect in the gaze direction contralateral to the recessed MR.

\section{Acknowledgments}

Funding/Support: This study was supported by National Eye Institute grant EY08313 from the US Public Health Service, which funded the design and conduct of the study; collection, management, analysis, and interpretation of the data; and preparation, review, or approval of the article. Dr Chaudhuri's work is supported by the Better Opportunities for Young Scientists in Chosen Areas of Science and Technology Fellowship of the Department of Science and Technology of the government of India. Dr Demer's work is supported by a Walt and Lilly Disney Award for Amblyopia Research from Research to Prevent Blindness, New York, New York, and The Shaw Family Endowment Fund.

\section{References}

1. Jampolsky A. Ocular divergence mechanisms. Trans Am Ophthalmol Soc. 1970; 68:730-822. [PubMed: 5524219]

2. Schanzer B, Bordaberry M, Jeffery AR, McNeil DE, Phillips PC. The child with divergence paresis. Surv Ophthalmol. 1998; 42(6):571-576. [PubMed: 9635905]

3. Nardi M, Dal Pozzo G, Romani A, Barca L. Divergence pseudoparalysis: a case report. Graefes Arch Clin Exp Ophthalmol. 1986; 224(4):371-373. [PubMed: 3710192]

4. Okudera H, Saito J, Matsuo T. A case of divergence palsy associated with bilateral chronic subdural hematoma [in Japanese]. No To Shinkei. 1991; 43(2):183-186. [PubMed: 1873087]

5. Lim L, Rosenbaum AL, Demer JL. Saccadic velocity analysis in patients with divergence paralysis. J Pediatr Ophthalmol Strabismus. 1995; 32(2):76-81. [PubMed: 7629673]

6. Stangler-Zuschrott E. Convergent strabismus in the age of presbyopia (author's transl) [in German]. Klin Monbl Augenheilkd. 1976; 168(6):775-783. [PubMed: 994382]

7. Bothun ED, Archer SM. Bilateral medial rectus muscle recession for divergence insufficiency pattern esotropia. J AAPOS. 2005; 9(1):3-6. [PubMed: 15729272]

8. Rutar T, Demer JL. "Heavy Eye" syndrome in the absence of high myopia: a connective tissue degeneration in elderly strabismic patients. J AAPOS. 2009; 13 (1):36-44. [PubMed: 18930668]

9. Hoover DL, Giangiacomo J. Results of a single lateral rectus resection for divergence and partial sixth nerve paralysis. J Pediatr Ophthalmol Strabismus. 1993; 30(2):124-126. [PubMed: 8501617]

10. Durnian JM, Maddula S, Marsh IB. Treatment of "heavy eye syndrome" using simple loop myopexy. J AAPOS. 2010; 14(1):39-41. [PubMed: 20227621]

11. Thacker NM, Velez FG, Bhola R, Britt MT, Rosenbaum AL. Lateral rectus resections in divergence palsy: results of long-term follow-up. J AAPOS. 2005; 9 (1):7-11. [PubMed: 15729273]

12. Ahadzadeghan I, Akbari MR, Ameri A, Anvari F, Jafari AK, Rajabi MT. Muscle belly union for treatment of myopic strabismus fixus. Strabismus. 2009; 17(2):57-62. [PubMed: 19551560]

13. Clark RA, Miller JM, Demer JL. Location and stability of rectus muscle pulleys: muscle paths as a function of gaze. Invest Ophthalmol Vis Sci. 1997; 38(1):227-240. [PubMed: 9008649]

14. Clark RA, Miller JM, Rosenbaum AL, Demer JL. Heterotopic muscle pulleys or oblique muscle dysfunction? J AAPOS. 1998; 2(1):17-25. [PubMed: 10532362]

15. Archer SM. The effect of medial versus lateral rectus muscle surgery on distance-near incomitance. J AAPOS. 2009; 13(1):20-26. [PubMed: 19131260] 
16. Fang ZT, Keyes MA. A novel mixture of propofol, alfentanil, and lidocaine for regional block with monitored anesthesia care in ophthalmic surgery. J Clin Anesth. 2006; 18(2):114-117. [PubMed: 16563328]

17. Tejedor J, Ogallar C, Rodríguez JM. Surgery for esotropia under topical anesthesia. Ophthalmology. 2010; 117(10):1883-1888. [PubMed: 20570361]

18. Yi JH, Chung SA, Chang YH, Lee JB. Practical aspects and efficacy of intraoperative adjustment in concomitant horizontal strabismus surgery. J Pediatr Ophthalmol Strabismus. 2011; 48(2):8589. [PubMed: 20506962]

19. Parks, MM.; Mitchell, PR.; Wheeler, MB. Concomitant Esodeviations. In: Tasman, W.; Jaeger, EA., editors. Duane's Foundations of Clinical Ophthalmology. Vol. 1. Philadelphia, Pennsylvania: Lippincott Williams and Wilkins; 2002. p. 12

20. Schroeder B, Krzizok T, Traupe H. Magnetic resonance imaging of the right extraocular muscle paths in healthy persons and in patients with high myopia [in German]. Rofo. 1998; 168(5):466473. [PubMed: 9617363]

21. Stern RM, Tomsak RL. Magnetic resonance images in a case of "divergence paralysis". Surv Ophthalmol. 1986; 30(6):397-401. [PubMed: 3726750]

22. Clark RA, Demer JL. Effect of aging on human rectus extraocular muscle paths demonstrated by magnetic resonance imaging. Am J Ophthalmol. 2002; 134 (6):872-878. [PubMed: 12470756]

23. Krzizok T, Kaufmann H, Traupe H. Nuclear magnetic resonance tomography diagnosis of eye motility disorder in high-grade myopia for planning an eye muscle operation [in German]. Ophthalmologe. 1997; 94(12):907-913. [PubMed: 9487762]

24. Wong I, Leo SW, Khoo BK. Loop myopexy for treatment of myopic strabismus fixus. J AAPOS. 2005; 9(6):589-591. [PubMed: 16414529]

25. Webb H, Lee J. Acquired distance esotropia associated with myopia. Strabismus. 2004; 12(3):149155. [PubMed: 15370522]

26. Thomas AH. Divergence insufficiency. J AAPOS. 2000; 4(6):359-361. [PubMed: 11124671]

27. Moore S, Harbison JW, Stockbridge L. Divergence insufficiency. Am Orthopt J. 1971; 21:59-63. [PubMed: 5089866]

28. Mojon DS. A modified technique for rectus muscle plication in minimally invasive strabismus surgery. Ophthalmologica. 2010; 224(4):236-242. [PubMed: 19940531]

29. Esser J, Schmitz F, Eckstein A. Eye muscle surgery in unilateral abducens palsy [in German]. Klin Monbl Augenheilkd. 2009; 226(10):812-817. [PubMed: 19830637]

30. Krzizok TH, Kaufmann H, Traupe H. New approach in strabismus surgery in high myopia. Br J Ophthalmol. 1997; 81(8):625-630. [PubMed: 9349146] 


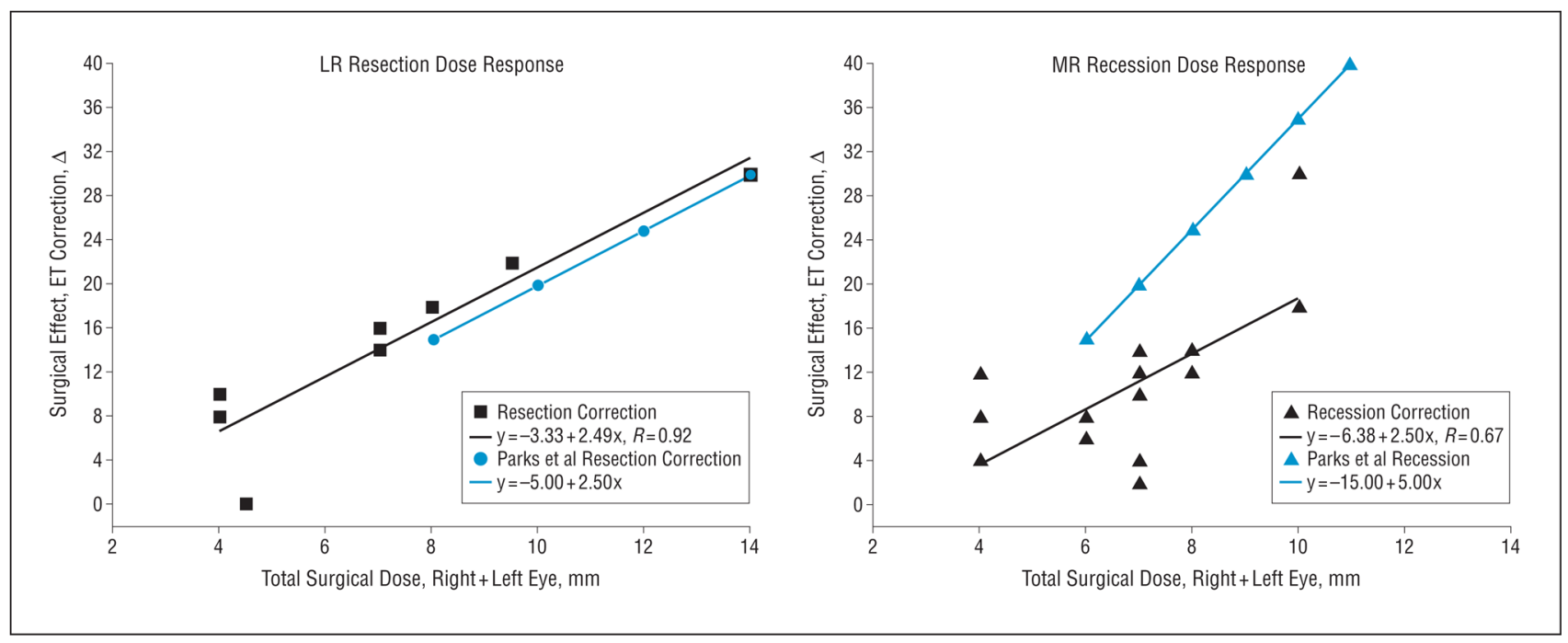

Figure.

Surgical dose-response effect for total binocular lateral rectus (LR) resection (left) or medial rectus (MR) recession (right) in divergence paralysis esotropia. The black symbols and linear regression fits indicate observed data for LR resection (squares) and MR recession (triangles), while corresponding blue symbols and fits indicate recommended values from surgical dose recommendations of Parks et al. ${ }^{19}$ Note that except for the published recommendations for MR recession with a slope of $5.00 \Delta / \mathrm{mm}$, the observed slopes for both LR resection and MR recession and recommended LR resection are almost precisely half of this value at about $2.50 \Delta / \mathrm{mm}$. This means that the incremental surgical response of divergence paralysis esotropia to MR recession is half that recommended by Parks et al for esotropia (ET) generally. ${ }^{19} \Delta$ indicates prism diopter. 


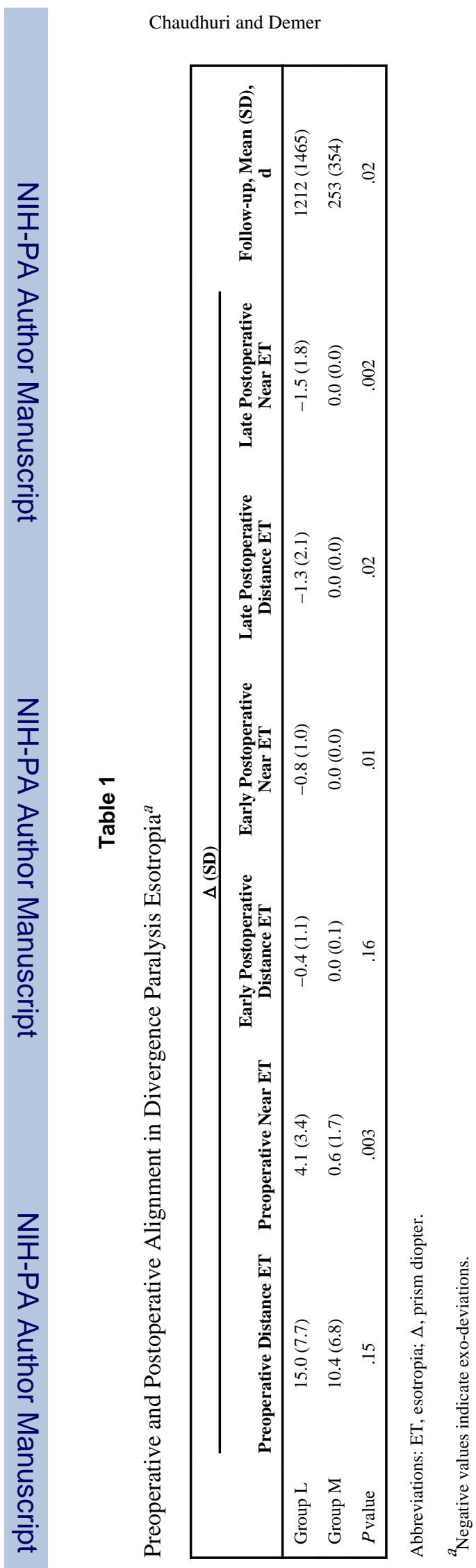

Page 9 
Table 2

Surgical Recommendation for MR Recession in DPE

\begin{tabular}{|cll|}
\hline Distance Esotropia, $\boldsymbol{\Delta}$ & MR Recession for Typical Esotropia, $\mathbf{~ m m}^{\boldsymbol{a}}$ & Current Recommended Bilateral MR Recession for DPE, $\mathbf{m m}$ \\
\hline 7.5 & & 3 \\
10 & & 3.5 \\
15 & 3 & 4.5 \\
20 & 3.5 & 5.5 \\
25 & 4 & 6 \\
30 & 4.5 & \\
35 & 5 & \\
40 & 5.5 & \\
\hline
\end{tabular}

Abbreviations: DPE, divergence paralysis esotropia; MR, medial rectus; $\Delta$, prism diopter.

a Recommendation of Parks et al. 19 